\title{
The Strengths and Weaknesses of Research Methodology: Comparison and Complimentary between Qualitative and Quantitative Approaches
}

\author{
Looi Theam Choy \\ (Faculty of Arts and Social Science, University of Malaya, Malaysia)
}

\begin{abstract}
The purpose of this study is compared strengths and weaknesses of qualitative and quantitative research methodologies in social science fields. Reviewed recent secondary resources, there is no best approach between both research methodologies due to existing strengths and weaknesses among both types of research methodologies. To plan and implement a research, choosing either one research methodology will still be occurred strengths and weaknesses for the research. This common study only based on existing secondary data and sources to interpret results. No primary data approach on this study due to sufficient source and data from secondary resources. The defined cases have proven that complementary approach between qualitative and quantitative approaches for a same research topic may provide expected results as both references better than just thoroughly considering either one research methodology approach.
\end{abstract}

Keywords: Qualitative Approach, Quantitative Approach, Qualitative Research Methodology, Quantitative Research Methodology.

\section{Introduction}

1.1 Introduction of Quantitative Approach in Research Methodologies

The process of conducting a quantitative study begins with a researcher selecting a topic. Quantitative researchers typically start with a general area of study or issue of professional or personal interest. Researchers must narrow it down to, or focus on, a specific research question that can be addressed in the study. Often this requires a careful review of the research literatures and developing hypotheses that frequently come from social theory (Neuman, 2006: 14).

Designing the study requires making decisions about the type of case or samples to select how to measure relevant factors and what research techniques such as questionnaires or experiments to be employed (Neuman, 2006: 14). In addition, quantitative methods characteristically refer to standardized questionnaires that are administered to individuals or households, which are identified through various forms of sampling usually random sampling (Dudwick, Kuehnast, Jones and Woolcock, 2006: 3). Basically, probability or non-probability sampling approaches are required researchers to choose a proper way before drawing a sample size.

Next is the data collection step. A quantitative researcher will very carefully record and verify information, almost always in one form of numbers and usually transfer the data into computer-readable format (Neuman, 2006: 14). Quantitative data can help establish correlations between given variables and outcomes. Such data should allow others to validate original findings by independently replicating the analysis (Dudwick, Kuehnast, Jones and Woolcock, 2006: 3). After that, analyze data step will be replaced. Often the research ends up with a large quantity of computer-generated output that provides the researcher next has to give meaning to or interpret the data. To draw a theory, quantitative researchers have to look at the analyzed data, using background knowledge on the research topic and questions. A researcher also considers alternative interpretation of the data compares the results of the study with previous studies and draws out its wider implications. The final step is to inform others means writing a report in a specific format for the study (Neuman, 2006: 14)

\subsection{Introduction of Qualitative Approach in Research Methodologies}

Qualitative researchers begin with a self-assessment and reflections about themselves as situated in a social-historical context. It is a highly self-aware acknowledgement of social self, or of a researcher's position in society. This type of approach does not narrowly focus on a specific question but ponder the theoretical philosophical paradigm in an inquisitive, open-ended settling in proves as they adopt a perspective (Neuman, 2006: 15). And also, qualitative methods typically refer to a range of data collection and analysis techniques that use purposive sampling and semi-structured, open-ended interviews (Dudwick, Kuehnast, Jones and Woolcock, 2006: 3). 
A qualitative researcher also will design a study, collect data, analyze data and interpret data same like a quantitative researcher does. He or she also tends to build new theory as well as draw on existing theory during these steps. At the interpret data stage, he or she creates new concepts and emphasize constructing theoretical interpretations (Neuman, 2006: 15).

At last, the final step called informs others but the report styles to present results to other people vary by approach (Neuman, 2006: 15).

\subsection{Background to the Study Problems}

Qualitative researchers often rely on interpretive or critical social science. They apply "logic in practice" and follow a nonlinear research path. They emphasize conducting detailed examinations of cases that arise in the natural flow of social life. In contrast, most of the quantitative researchers rely on a positivists approach to social science. They apply "reconstructed logic", and follow a linear research path. Quantitative researchers emphasize precisely measuring variables and testing hypotheses that are linked to general causal explanation (Nueman, 2006: 151).

One of the differences between the two styles comes from the nature of the data. Soft data, in the form of impressions, words, sentences, photos, symbols and so forth, dictate different research strategies and data collection techniques than hard data, in the form of numbers. Other differences are different assumptions about social life and different objectives. The differences can make tools used by the other style inappropriate or irrelevant. People who judge qualitative research by standards of quantitative research are often disappointed, and vice-versa (Neuman, 2006: 151).

Researchers who use one style alone do not always communicate well with those using the other, but the languages and orientations of the styles are mutually intelligible. It takes time and effort to understand both styles and to see how they can be complementary (Neuman, 2006: 151).

The historically negative bias against qualitative research is discussed, as well as the strengths and weaknesses of both approaches, with issues highlighted by reference to nursing research. Consideration is given to issues of sampling, the relationship between the researcher and subject, methodologies and collated data, validity, reliability, and ethical dilemmas (Carr, 1994: 716). For other potential biases in data collection procedures, there may be no clear advantage to either qualitative or quantitative approaches, although qualitative research methods have the advantage that they may be able to identify and handle response bias as it occurs, rather than afterwards. For example, biases due to the respondent's 'mental set' refer to the way that perceptions based on previous items influence replies to later ones. In the social desirability bias, a respondent replies so as to appear socially acceptable in eyes of interviewer. Acquiescence bias, similarly, covers responses that are influenced by the respondent's perception of what would be desirable to the researcher, especially to an influential one (Mcdowell and Maclean, 1998: 19).

\subsection{Objectives of the Study}

This study is purposely and merely compared the strengths and weaknesses of quantitative and qualitative research methodologies in social science fields. Evaluation among the strengths and weaknesses of quantitative and qualitative research methodologies also the objective for this study in the field of social science. Identification of complementary between both qualitative and quantitative approaches also the intention for this study.

\subsection{Significance of the Study}

To identify the strengths and weaknesses of both qualitative and quantitative research methodologies are appropriated paths for researchers selecting their research methodologies according to their specific topics. Thus, researchers can decide their research methodologies referring to this evaluation. In addition, complementary approach between both qualitative and quantitative research methodologies can provide better results. Hence, researchers can apply this approach as alternative solution for their researches especially in social science field.

\subsection{Scope of the Study}

The contents of this scope only focus on the evaluation and comparison between strengths and weaknesses of research methodologies in social science, quantitative and qualitative approaches. The time scope for this study commenced in March 2014 within one week to complete all of it.

\subsection{Theoretical Framework}

The illustrated diagram "Fig. 1" is the entire description of strengths and weaknesses of qualitative and quantitative research methodologies. The left side and right side are consisting specific strengths and weaknesses of both qualitative and quantitative research methodology approaches. The middle of qualitative and 
quantitative research methodology approaches are top-down connected to indicate the better research results for a single research can be achieved through the complementary approach by both qualitative and quantitative approaches but not combining the process of both research methodologies into a single research. Furthermore, the top-down connection also advocated the both research results are totally based on separation of both qualitative and quantitative research methodologies, just only comparing both of the research results as references under a same research topic. The outline of the theoretical framework has illustrated through below diagrams:

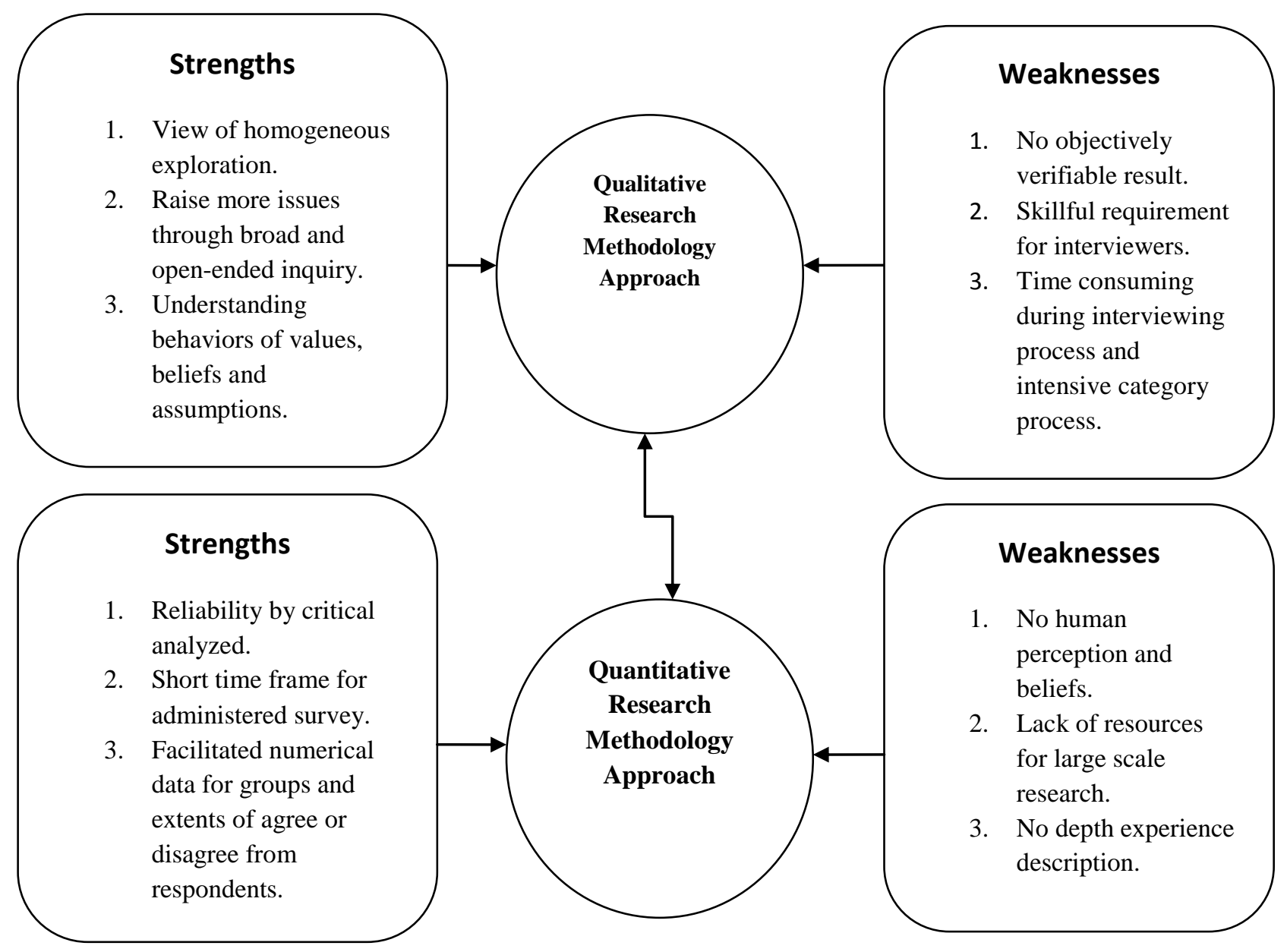

"Figure 1" The model of strengths and weaknesses of qualitative and quantitative research methodologies Source: owned by author.

\subsection{Hypotheses}

H0: There is no strength enhancement and weakness reduction for complementary between both qualitative and quantitative research methodologies under a same research.

H1: There is a strength enhancement and weakness reduction for complementary between both qualitative and quantitative research methodologies under a same research.

\subsection{Research Question}

Comparatively evaluate the strengths and weaknesses of quantitative and qualitative research methodologies.

\subsection{The Strengths of Quantitative Research Methodology}

\section{Literature Reviews}

The quantitative as survey approach has two significant advantages. First, it can be administered and evaluated quickly. There is no need to spend time at the organization prior to administering the survey, and the responses can be tabulated within a short timeframe. Second, numerical data obtained through this approach facilitates comparisons between organizations or groups, as well as allowing determination of the extent of agreement or disagreement between respondents (Yauch and Steudel, 2003: 473). 
The advantage of legitimate quantitative data, that is data which is collected rigorously, using the appropriate methods and analysed critically, is in its reliability (ACAPS, 2012: 6).

\subsection{The Weaknesses of Quantitative Research Methodology}

The strengths of quantitative research can, however, also be weaknesses. Many important characteristics of people and communities including both rich and poor, for example, identities, perceptions, and beliefs that cannot be meaningfully reduced to numbers or adequately understood without reference to the local context in which people live (Dudwick, Kuehnast, Jones and Woolcock, 2006: 3).

Effective quantitative research usually requires a large sample size sometimes several thousand households. However, lack of resources sometimes makes large-scale research of this kind impossible. In many settings particularly developing countries, interested parties (e.g., governments, nongovernmental organizations and public service providers) may lack the skills and especially the resources needed to conduct a thorough quantitative evaluation (Dudwick, Kuehnast, Jones and Woolcock, 2006: 3).

In term of disaster survey, the shortcoming of quantitative data is that it fails to provide an in depth description of the experience of the disaster upon the affected population. Knowing how many people are affected and their locations does not provide sufficient information to guide agencies and sectors on what they should plan for in terms of response (ACAPS, 2012: 6).

\subsection{The Strengths of Qualitative Research Methodology}

Qualitative methods that allow researchers to explore the views of homogenous as well as diverse groups of people help unpack these differing perspectives within a community. Because social capital is relational - it exists between people - asking a group of people to respond together to certain questions and hypothetical situations may yield information that is more nuanced than data derived from surveys (Dudwick, Kuehnast, Jones and Woolcock, 2006: 3).

The primary strength of the qualitative approach to cultural assessment is the ability to probe for underlying values, beliefs, and assumptions. To gain a full appreciation of an organization, it is necessary to understand what is driving their behavior (Yauch and Steudel, 2003: 472).

The other great benefit with a qualitative approach is that the inquiry is broad and open-ended, allowing the participants to raise issues that matter most to them. The qualitative researcher typically does not have a preconceived, finite set of issues to examine (Yauch and Steudel, 2003: 472).

\subsection{The Weaknesses of Qualitative Research Methodology}

The major drawbacks associated with qualitative cultural analysis are firstly, the process is time-consuming, and secondly, a particular, important issue could be overlooked. The second potential problem is that a particular issue could go unnoticed. All researchers' interpretations are limited. As positioned subjects, personal experience and knowledge influence the observations and conclusions. Also, because qualitative inquiry is generally open-ended, the participants have more control over the content of the data collected (Yauch and Steudel, 2003: 472-473).

ACAPS's supporting document states the weaknesses inherent in qualitative data are that it, firstly, results in data which is not objectively verifiable. Secondly, it requires a labour intensive analysis process such as categorization, recoding, etc. and thirdly, it needs skilled interviewers to successfully carry out the primary data collection activities (ACAPS, 2012: 10).

\section{Materials and Methodologies}

This part is the outline of the methods to implement and perform the research. For this study, the secondary data or source is the main focus and intentions to discover and analyze the strengths and weaknesses of research methodologies, qualitative and quantitative approaches. Those secondary data could be discovered by related text books, online journal articles and online supporting documents. Apparently, case study, contents or context analysis is the primary method to analyze the strengths and weaknesses of research methodologies on qualitative and quantitative approaches. Due to this study as generic or common research, the secondary data or source is sufficiently to justify the research purposes or targets without primary data approach.

\section{Findings and Results}

\subsection{Case 1: Qualitative Case Study Method at an Information System Organization}

The case study approach refers to a group of methods which emphasize qualitative analysis. Data are collected from a small number of organizations through methods such as participant-observation, in-depth interviews, and longitudinal studies. The case study approach seeks to understand the problem being investigated. It provides the opportunity to ask penetrating questions and to capture the richness of 
organizational behavior, but the conclusions drawn may be specific to the particular organizations studied and may not be generalizable (Gable, 1994: 2).

Fervent critics of qualitative methods are many, and hail largely from the physical sciences. More objective criticism has come from the social sciences. Kerlinger, an author identifies three major weaknesses of qualitative research, the inability to manipulate independent variables, the risk of improper interpretation, and the lack of power to randomize. Another author, Lee identifies four corresponding problems with case study research, a lack of controllability, deductibility, repeatability and generalizability, where the latter two limitations stem largely from the aforementioned lack of power to randomize (Gable, 1994: 2). No doubts, putting a side quantitative approach could not generate a satisfy results for all of them. Hence, the results of quantitative approach are necessary that allowing them to compare the results as references between both research methodologies.

For qualitative approach, the author, Benbasat and his group mates identify three strengths of case study research in information systems: (1) the researcher can study information systems in a natural setting, learn about the state of the art, and generate theories from practice; (2) the method allows the researcher to understand the nature and complexity of the process taking place; and (3) valuable insights can be gained into new topics emerging in the rapidly changing information systems field. Another author, Yin suggests that case studies are appropriate where the objective is to study contemporary events, and where it is not necessary to control behavioral events or variables. Yin further suggests single case studies are appropriate if the objective of the research is to explore a previously un-researched subject, whereas multiple-case designs are desirable when the intent of the research is description, theory building, or theory testing. Benbasat aand his group mates suggest that multiple-case designs allow for cross case analysis and the extension of theory. The author, Van Maanen states, "... no matter what the topic of study, qualitative researchers, in contrast to their quantitative colleagues, claim forcefully to know relatively little about what a given piece of observed behavior means until they have developed a description of the context in which the behavior takes place and have attempted to see the behavior from the position of its originator (Gable, 1994: 2).

\subsection{Case 2: Quantitative Survey in Pakistan Conflict}

In Pakistan in 2008 during large scale conflict related displacement, the child protection cluster wanted to know numbers of separated children in each site visited. The sites comprised a small, purposive sample of all of sites in the area and varied significantly in population size and composition (ACAPS, 2012: 7).

Questions were asked to community groups about numbers of separated children. Asking for this information was taxing for the community groups to answer, and field teams found that male and female groups gave vastly different numeric answers to the question making it both impossible to resolve for each site and resulting in un-analyzable information (ACAPS, 2012: 7). Obviously, sample size should be enlarged during this case due to statistically results would be more reliable if sample size large enough. In addition, perceptions and beliefs might vary between male and female. Hence, a qualitative research is necessary for complementary of quantitative research.

While it would have been useful to know whether a trend of unaccompanied children resulted from the initial displacement, reliable data on numbers of unaccompanied children could not be generalized from the data, rendering the exercise of collecting this specific piece of data meaningless and wasteful of both time and resources (ACAPS, 2012: 7).

\subsection{Case 3: Haiti, Quantitative Survey on RINAH Disaster}

In previous multi cluster needs assessments, pressure from agencies to include a multitude of sector specific questions in quantitatively framed questionnaire tools resulted in assessment teams collecting quantitative data that was neither reliable nor analyzable and thus unusable. In the Haiti RINAH, for example, 190 questions were included in the questionnaire, but out of the 76 that were usable and reliable for inclusion in the final RINAH report, none were quantitative (ACAPS, 2012: 6). Indeed, proper questionnaires such as reliability and validity factors should be proven before dispatching for those respondents. Moreover, the case might be added open-ended questions such as comments could provide qualitative data for analyzing process but not suitable for quantitative approach.

\subsection{Case 4: Qualitative Approach shaped the Political Science Program at Truman}

Quantitative measures like national exams and surveys force students to give standardized responses to uniform questions and produce data that is easily manipulated and compared. Qualitative exit interviews have allowed the faculty to delve deeper into the reasons behind student attitudes and to probe them with a depth and breadth not possible in quantitative surveys. This benefit echoes the literature of qualitative methods discussed earlier. Also, students see the interview as a nice counter to completion of anonymous and impersonal surveys. On an affective level qualitative interviews give students face-to-face contact with faculty who care about their 
personal well-being and opinions. It is not uncommon for students to generate questions for the faculty, or for both sets of participants to engage in informal exchanges. The power of this human interaction can be strong. Perhaps more importantly faculty members receive feedback face to face from the students themselves (Young and Hagerty, 2007: 8). Unsatisfied quantitative results led the Truman's researchers transforming quantitative approach to qualitative approach that could understand student's perceptions and motivations.

\section{Conclusion}

No matter which types of research methodologies, qualitative or quantitative approach has been selected for research purposely; critics, debates or comments are still appeared between specialists of qualitative and quantitative approaches. Kindly refer to the above mentioned cases, choosing either one type of research methodology has still embraced negative and positive impacts of the results and output. Thus, it could be concluded that there is no perfect between qualitative and quantitative research methodologies. The strengths and weaknesses of both types of research methodologies will still be performed, anticipated and shown for further social science researches. The above mentioned cases also shown that complementary between both qualitative and quantitative research methodologies could provide better solutions. A comparison and complimentary results as references from both separated processes on qualitative and quantitative approaches in a same research topic may reduce or perhaps eliminate those limitations and bias. This is the alternative way to provide better solution in research methodologies.

\section{Acknowledgements}

I would wish to express my appreciation and gratitude to my father, Mr. Looi Yon Cheong and my mother, Mrs. Chong Lan currently both living in Ipoh state, Malaysia, who always provide me with the encouragement, support and understanding throughout my life.

\section{References}

[1]. ACAPS (2012) Qualitative and Quantitative Research Techniques for Humanitarian Needs Assessment.

[2]. Carr, L. T. (1994) The Strengths and Weaknesses of Quantitative and Qualitative Research: What Method for Nursing, Journal of Advanced Nursing, No.20, pp. 716-721.

[3]. Dudwick, N., Kuehnast, K., Jones, V. N., and Woolcock, M. (2006) Analyzing Social Capital in Context: A Guide to Using Qualitative Methods and Data, World Bank Institute, Washington.

[4]. Gable, G. G. (1994) Integrating Case Study and Survey Research Methods: An Example in Information Systems, European Journal of Information Systems, Vol. 3, No. 2, pp.112-126, URL Accessible: http://eprints.qut.edu.au

[5]. Mcdowell, I. and Maclean, L. (1998) Blending qualitative and quantitative study methods in health services research, Health Informatics Journal No.4, pp.15-22.

[6]. Neuman, W. L. (2006) Social Research Methods: Qualitative and Quantitative Approaches $6^{\text {th }}$ Edition, Pearson International Edition, USA.

[7]. Yauch, C. A. and Steudel, H. J. (2003) Complementary Use of Qualitative and Quantitative Cultural Assessment Methods, Organizational Research Methods, Vol. 6, No. 4, pp. 465-481.

[8]. Young, C. and Hagerty, R. (2007) Blending Qualitative and Quantitative Methods for Program Evaluation: The Application and Insights of the Exit Interview, 4th Annual Meeting of the American Political Science Association Teaching and Learning Conference, Charlotte. 